\section{Magnetic resonance imaging - Where is it going to take us?}

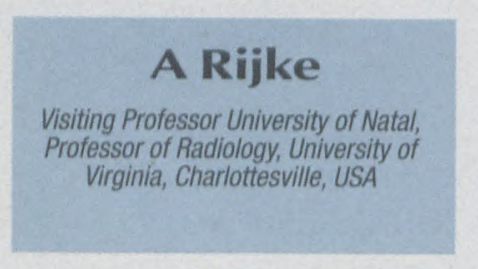

- and I am one of them - that MRI, particularly that of the CNS and the musculoskeletal system holds the ultimate capability of uncovering any morphology or pathology needed to direct therapeutic approach. But will it?

Within a decade of its clinical introduction MRI has become the imaging test of choice for meniscal pathology of the knee. Today, MRI of the menisci is one of the most frequent uses of the technology showing the fibrocartilagic as well as muscle, tendon, and ligament pathology in exquisite detail reliably dictating therapy and directing the surgeon to the site of injury. But does it?

MRI has emerged as the leading imaging modality for diagnosing aseptic necrosis, evaluating soft tissue masses and staging tumours in the wrist and ankle. Ongoing improvements in surface coil design have rendered MRI the most powerful tool for imaging structural abnormalities of the elbow and shoulder including numerous disorders that may clinically mimic pathology of the lateral epicondyle and rotator cuff. For instance, unsuspected ruptures of the lateral ulnar collateral ligament (LUCL) or its iatrogenic tearing secondary overaggressive release of the common extensor tendon, can be visualised on MRI and point to subtle posterolateral elbow destabilisation. But should it?

The answers to these queries are, of course, anybody's guess, but it is worth taking a closer look. They will, after all, affect us all by determining nothing less than the future of our speciality. First then, technological advancement, particularly in the software sector, in the next 10 years or so is likely to further imaging capability cal followers. We all strongly believe 


\section{SIEMENS}

\section{SONOLINE} The benchmark in ultrasound

Siemens new ultrasound family breaks new ground in ultrasound technology. Designed in collaboration with clinicians, the SONOLINE Prima, Versa, and Elegra features state-of-the-art digital electronics making higher levels of imaging performance possible.

\section{SONOLINE Prima}

The highest performing system in its class, the Prima integrates innovation and power in a compact and mobile design.

\section{SONOLINE Versa Pro}

Offers the widest variety of applications and performance levels and with its innovative design it allows you to expand and upgrade as your needs grow.

\section{SONOLINE Elegra}

Technological advantages that have never before existed in a single system have been combined in the Elegra, to produce image quality that exceeds the imaginable.

The platform technology utilised in the SONOLINE generation makes software upgrades simple, thereby protecting your investment as your business grows. All the products in the range can be networked, making telemedicine solutions possible and affordable for both urban and rural environments.

Qualified technicians will ensure you of the best service and support, while Siemens further meets your requirement by offering finance/ leasing packages and comprehensive insurance at an excellent rate.

For more information, contact us on:

Tel: (011) 652-2383

Fax: (011) 652-2322

\section{d

.

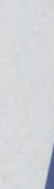

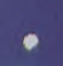

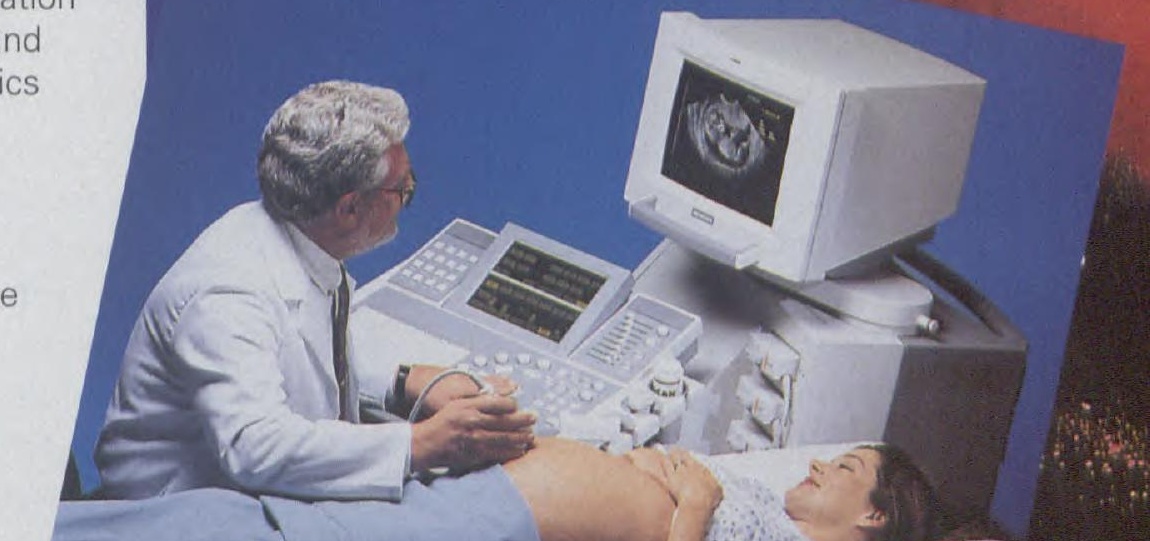




\section{from page 6}

with emphasis on diagnostic and pathological criteria.

It is, however, far less likely to dictate clinical strategy. There are several reasons for this. One of them is related to the inherent shortcomings of MRI, some of which are euphemistically referred to as pitfalls, wrongly suggesting that they can be avoided if one is aware of them. Other, more important reasons are to be found in the area of health care delivery economics, patient population base/dynamics and of who will be the key players in the future. It is interesting to note in this context, that in the last five years, MRI equipment manufacturers have spent a significant part of their research dollar on the development of low-field permanent magnet systems as part of both open-configuration and dedicated extremity scanners. Although only two such systems have so far been approved by the United States Food and Drug Administration and only a few are in actual clinical use today, more are sure to enter the market place soon. They offer a number of practical and economic advantages including increased patient comfort and risk at much lower cost. It is wise to take note of manufacturers' thinking because they have a keen eye on future potential and development. Targeting clinicians in private practice with affordable permanent magnet equipment is definitely on their short-list agenda. And that will set the stage for who will image what, in whom and for whom.

Yet another reason why MRI technology is unlikely to dictate clinical approach is the concurrent technological development in other areas of diagnostic expertise. Arthroscopy is a case in point: in the same time period that MR imaging of the internally deranged knee approached near-perfection, the arthroscope has seen ever so much development in fibroscopic and manipulative sophistication. Add to this the capability of intra-articular excision and reconstruction and you can see why - economic considerations aside - no orthopaedic surgeon would ever let go of this tool, regardless of what the MR radiologist may have to offer.

If MRI by itself does not dictate therapy, it will surely help to direct the arthroscopist to the site of suspected injury. Well, no, not in practice, that is: for some anatomical reasons that are not coincidental, those regions of the knee joint that are most difficult to visualise by arthroscopy are also the most difficult to image. It is only small consolation that injury to the posterolateral aspect of the lateral meniscus rarely needs treatment.

The superb imaging capability of MRI is often the cause of discussion with clinicians about findings that may or may not relate to the patient's symptoms. One classical example is spinal stenosis with MRI showing details of spinal canal bony dimensions and soft tissue alike, often prompting surgeons to presumptive conclusions. Another is the continuity of the LUCL in relation to subtle posterolateral instability. While it is the radiologist's duty to comment on the integrity of the LUCL in the context of postoperative elbow imaging, it is clearly the surgeon's preoperative understanding of lateral elbow stabilisers that is required to prevent accidental disruption of this ligament. In fact, the orthopaedic literature had documented the significance of the LUCL in elbow stability well before this ligament attracted the attention of MR radiologists.
So with the technological future of MRI including three-dimensional rendering capability foreseeable, the actual clinical role and how that is going to be played out and by whom is far less predictable. One scenario foreshadows MR radiologists to be increasingly involved in the departments of their subspeciality to the point of joining their faculty/staff as the imaging specialist. Likely candidates would be neuro-, musculoskeletal and cardiothoracic radiologists. Such developments, while possibly better serving the imaging requirements of clinical departments, would not fail to severely strain the organisational and administrative capabilities of radiology departments as we know them today.

\section{Interesting images}

At the suggestion of one of our readers, we will reserve space in the SA Journal of Radiology for the publication of interesting cases with the emphasis on images. The images are to be of excellent quality in order to be suitable for publication, and the publisher reserves the right to reject any which would not reproduce satisfactorily. Pathological pictures are also welcome.

A heading and a brief explanation, together with picture legends, should accompany the images.

\section{The Editor}

